\title{
Large Intestinal Anastomotic Leakage
}

National Cancer Institute

\section{Source}

National Cancer Institute. Large Intestinal Anastomotic Leakage. NCI Thesaurus. Code C78408.

Leakage due to breakdown of a large intestinal anastomosis. 